\title{
Propagation of Quantum Walks in Electric Fields
}

\author{
C. Cedzich, ${ }^{1}$ T. Rybár, ${ }^{1}$ A. H. Werner, ${ }^{2}$ A. Alberti, ${ }^{3}$ M. Genske, ${ }^{3}$ and R. F. Werner ${ }^{1}$ \\ ${ }^{1}$ Institut für Theoretische Physik, Leibniz Universität Hannover, Appelstrasse 2, 30167 Hannover, Germany \\ ${ }^{2}$ Dahlem Center for Complex Quantum Systems, Freie Universität Berlin, 14195 Berlin, Germany \\ ${ }^{3}$ Institut für Angewandte Physik, Universität Bonn, Wegelerstrasse 8, 53115 Bonn, Germany
}

(Received 11 February 2013; published 14 October 2013)

\begin{abstract}
We study one-dimensional quantum walks in a homogenous electric field. The field is given by a phase which depends linearly on position and is applied after each step. The long time propagation properties of this system, such as revivals, ballistic expansion, and Anderson localization, depend very sensitively on the value of the electric field, $\Phi$, e.g., on whether $\Phi /(2 \pi)$ is rational or irrational. We relate these properties to the continued fraction expansion of the field. When the field is given only with finite accuracy, the beginning of the expansion allows analogous conclusions about the behavior on finite time scales.
\end{abstract}

DOI: 10.1103/PhysRevLett.111.160601

PACS numbers: 05.60.Gg, 03.65.Db, 72.15.Rn

In this Letter we analyze the surprisingly rich longtime behavior of a simple quantum lattice system, a one dimensional discrete time quantum walk in a homogenous electric field. This system has recently been realized experimentally [1]. Here we provide the theoretical explanation for the phenomena observed in [1], covering also time scales much longer than those accessible by current-day experiments. The most remarkable feature of this system is that its longtime behavior depends dramatically on whether the key parameter, the electric field, is rational or irrational. This appears paradoxical, because the difference between these number classes would require infinite experimental precision. Yet, as reported in [1], a qualitative difference can be seen. We will give a very detailed resolution of this apparent paradox, showing exactly which features of the field have predictive value on which time scales, and how the precise nature of the field is reflected in the infinite time behavior.

Similar phenomena involving rational vs irrational parameters are known from systems such as the quantum kicked rotator $[2,3]$ and the propagation of quantum particles in quasiperiodic potentials [4]. Another famous case is the Hofstadter butterfly [5], which pertains to a two dimensional lattice system in a magnetic field. The crucial parameter in this case is the magnetic flux per unit cell. This system is analyzed in terms of the one dimensional Mathieu equation, whose variants have recently been analyzed with great success in terms of certain dynamical systems [6]. The crucial role of the continued fraction expansion [7] for our work echoes its role in the theory of chaotic dynamical systems (see, e.g., [8]), and also in the Shor algorithm [9], where it is used to identify a particular fraction.

Quantum walks have recently attracted much attention as a computational resource [10-15] as well as a model of discrete-time evolutions of single particles with internal degrees of freedom [16-20], which in particular provides a model to describe the energy transfer in light harvesting complexes [21-23]. In addition, it has been shown that quantum walks exhibit a rich variety of quantum effects such as Landau-Zener tunneling [24], the Klein paradox [25], topological phases [26,27], and Bloch oscillations [24] as well as the formation of molecules for two interacting particles each performing a quantum walk [28]. Furthermore, quantum walks have been experimentally realized in such diverse physical systems as neutral atoms in optical lattices [29], trapped ions [30,31], wave guide lattices [32,33] and light pulses in optical fibers [34,35] as well as single photons in free space [36].

Quantum walks in external "simulated" fields play a crucial role in the endeavor of building a quantum simulator for solid state models, in particular of magnetic systems such as Hubbard models [37,38]. They also allow the realization of nontrivial symmetry classes, for example, by breaking time reversal invariance. The recent classification of topological phases $[26,27]$ based on such symmetries shares with the present study the phenomenon of very flat bands in the dispersion relation of a walk.

Exactly the same system as ours has been studied mostly in the rational case, namely, numerically in $[39,40]$ and by exact diagonalization on a finite ring [41]. In the irrational case Anderson localization [42-44] is conjectured in [41]. We agree that this is possibly the typical case assuming the crucial parameter, here referred to as the electrical field, $\Phi$ to be a uniformly distributed random number, but we also exhibit irrational cases with a quite different behavior. Most extremely, we show the possibility of "hierarchical motion," which is characterized by an infinite hierarchy of time scales in which ever more perfect revivals alternate with ever larger ballistic excursions.

An overview of the qualitatively different infinite time behaviors is given in Table I. Some of the terminology will be explained in detail in the respective sections. The quasienergy spectrum refers to the spectral properties of the 
TABLE I. Overview: The connection between the properties of $\Phi$, its continued fraction coefficients $c_{i}$, propagation, and the spectrum of the unitary evolution operator. Detailed explanations are found in the subsections for the respective columns.

\begin{tabular}{llll}
\hline \hline & \multicolumn{1}{c}{ Rational } & Almost rational & Very irrational \\
\hline Continued fraction expansion & Terminates & $c_{i} \rightarrow \infty$ rapidly & $c_{i}$ bounded \\
Propagation & Ballistic with revivals & Hierarchical & Localized, almost periodic \\
Quasienergy spectrum & Absolutely continuous & Singular continuous & Dense set of eigenvalues \\
Status & Proved & Proved & Numerical evidence \\
\hline \hline
\end{tabular}

unitary walk operator in the sense of, e.g., [45], Chap. VII.2. The properties in the table depend on the exact value of $\Phi$ or, equivalently, on its entire continued fraction representation. For an experimentally given field with finite accuracy, only an initial segment of this expansion can be reliably determined. Depending on how one continues the expansion, any of the three classes is compatible with such data. However, the behavior on a finite time scale can be reliably predicted from the data. As in the infinite case, the important input is the size of the continued fraction terms $c_{i}$, which, in particular, determine the quality of revivals as explained below.

Definition of the system.-For definiteness and to fix notations let us explicitly define the class of systems under investigation. We consider particles on the $1 \mathrm{D}$ lattice $\mathbb{Z}$ with a two dimensional space of internal degrees of freedom. Basis vectors are thus $|x, \alpha\rangle$ with $x \in \mathbb{Z}$ and $\alpha= \pm 1$. The state dependent shift is defined by $S|x, \alpha\rangle=$ $|x+\alpha, \alpha\rangle$. We consider, moreover, a coin operation $C|x, \alpha\rangle=\sum_{\beta} C_{\alpha \beta}|x, \beta\rangle$ with a fixed matrix $C \in \mathrm{SU}(2)$, i.e.,

$$
C=\left(\begin{array}{cc}
a & b \\
-\bar{b} & \bar{a}
\end{array}\right)
$$

with $|a|^{2}+|b|^{2}=1$. A standard case is the Hadamard walk with $a=b=1 / \sqrt{2}$. The third element will be a phase shift depending linearly on $x$, given by the operator $\exp (i \hat{x} \Phi)|x, \alpha\rangle=\exp (i x \Phi)|x, \alpha\rangle$. The constant $\Phi$ is referred to as the electric field. The overall time step is then given by

$$
W_{\Phi}=e^{i \hat{x} \Phi} C S \text {. }
$$

Rational approximations.-We will analyze the behavior of $W_{\Phi}$ and its iterations by comparison with similar walks $W_{\Phi^{\prime}}$, where $\Phi^{\prime}=2 \pi n / m$ is a rational approximation to $\Phi$. The basic tool is an estimate for the difference between two states evolved from the same initial state by walks with slightly different fields $\Phi$ and $\Phi^{\prime}$. As an initial state we choose a unit vector $\psi$ which is nonzero only for lattice sites $x$ with $|x|<L$. Then for one step $\| W_{\Phi} \psi-$ $W_{\Phi^{\prime}} \psi \| \leq \max _{|x| \leq L}\left|\exp (i x \Phi)-\exp \left(i x \Phi^{\prime}\right)\right| \leq L\left|\Phi-\Phi^{\prime}\right|$. After $t$ steps, adding the deviations, and observing that the localization region of $\psi$ increases by 1 in every step we get

$$
\left\|W_{\Phi}^{t} \psi-W_{\Phi^{\prime}}^{t} \psi\right\| \leq \frac{t}{2}(t+2 L-1)\left|\Phi-\Phi^{\prime}\right| .
$$

So, for example, if $\Phi^{\prime}$ approximates $\Phi$ to within $\varepsilon=10^{-4}$, and the particle starts at the origin $(L=1)$, we can use $W_{\Phi^{\prime}}^{t} \psi$ to predict the behavior of $W_{\Phi}^{t} \psi$ for up to $\varepsilon^{-1 / 2} \approx$ 100 steps. The square root makes decimal approximations (or approximations in any other digital base) quite useless. Instead, one can look for denominators $m$ such that the approximation error is not of order $m^{-1}$, as can always be trivially achieved, but of order $m^{-2}$ or better.

The systematic way to generate such approximations is the so-called continued fraction expansion [7]. For a number $x=x_{0} \geq 0$ it is computed by taking the integer part $c_{0}=\left\lfloor x_{0}\right\rfloor$, setting $x_{1}=1 /\left(x-c_{0}\right)$, and repeating this process for $x_{1}$ to get $c_{1}$, etc. The number $x$ is then uniquely characterized by the sequence $\left(c_{0}, c_{1}, \ldots\right)$. One gets an explicit sequence of approximating rationals $n_{i} / m_{i}$, where the denominators $m_{i}$ and numerators $n_{i}$ both satisfy the recursion $r_{i}=c_{i} r_{i-1}+r_{i-2}$ with initial values $n_{0}=c_{0}$, $n_{-1}=1, m_{0}=1, m_{-1}=0$. This is equivalent to an iteration in which at step $i$ the denominator $c_{i}$ is replaced by $c_{i}+1 / c_{i+1}$, leading to the typographical nightmare from which the continued fractions derive their name. The desired approximation of $x$ of order $1 / m_{i}^{2}$ is stated as

$$
\left|x-\frac{n_{i}}{m_{i}}\right|<\frac{1}{c_{i+1} m_{i}^{2}} .
$$

Therefore, when $\Phi^{\prime}=2 \pi n_{i} / m_{i}$ is a continued fraction approximation of $\Phi$, the error in (3) is of order $\left(t / m_{i}\right)^{2}$, so the $i$ th approximation is valid roughly on the time scale of the denominator $m_{i}$, and correspondingly longer if the next term $c_{i+1}$ is large.

Intuitively, Eq. (4) says that large terms $c_{i}$ correspond to especially good approximations. For example, the continued fraction expansion of $\pi$ begins with $(3,7,15, \ldots)$ and the second approximant $\pi \approx 22 / 7$ has an error $<1 /(15 \times 49) \approx 10^{-3}$. In this sense the "most irrational number" is the golden ratio $\varphi=(1+\sqrt{5}) / 2$ with expansion $(1,1,1, \ldots)$.

The rational case: Revivals and ballistic expansion.Let us begin with the rational case, in which $\Phi=2 \pi n / m$, where it is understood that $n, m$ do not have common factors. The phase factor $\eta=\exp (i \Phi)$ is then a primitive $m$ th root of unity, i.e., $\eta^{m}=1$, but this holds for no smaller power. There are two ways to reduce the analysis to the case of standard, translationally invariant walks $[18,19,46]$. One is to use the fact that the field phase factor is periodic 
with period $m$, and hence always group $m$ sites together into a "supercell" of "coin dimension" $2^{m}$. This spatial regrouping is carried out in [1], giving a band structure with a $2^{m}$-branched dispersion relation. Here we will use instead a temporal regrouping, i.e., consider the operator $W_{\Phi}^{m}$, which likewise commutes with translations. This is a walk with two coin states, but allowing for steps over up to $m$ sites. The key fact about this walk is that, even for moderately large $m$, it hardly moves, in the sense that the walk operator is close to the identity operator (up to a phase). Phrased as a statement about the ungrouped walk $W_{\Phi}$, we get a revival of the initial state after $m$ steps. The precise statement is the following revival theorem:

$$
\begin{gathered}
\left\|W_{\Phi}^{2 m}+\mathbb{1}\right\|_{\text {op }}=2|a|^{m} \quad m \text { odd } \\
\left\|W_{\Phi}^{m}+(-1)^{m / 2} \mathbb{1}\right\|_{\text {op }}=2|a|^{m / 2} \quad m \text { even, }
\end{gathered}
$$

for $\Phi=2 \pi n / m$. Hence, for odd $m$ any initial state is reproduced after $2 m$ steps with accuracy (trace norm distance) $4|a|^{m}$, which is exponentially small in $m$, since $|a|<1$, except in trivial cases. For the Hadamard walk we get an error $2^{-m / 2}$. The different behavior of the even and odd cases is to be expected for coined walks, since the amplitude for no jump or a jump by an even distance is zero. Hence, the revival of a state localized at a point is only possible after an even number of steps.

The proof of the revival theorem is given in the Supplemental Material [47], and could also be based on the eigenvalues provided, without proof, in [41]. It yields also the dispersion relations for $W_{\Phi}^{m}$, concretely

$\cos \omega_{ \pm}(k)= \begin{cases}a^{m} \cos (m k) & m \text { odd } \\ -a^{m} \cos (m k)+(-1)^{m / 2+1}\left(1-|a|^{m}\right) & m \text { even. }\end{cases}$

The upshot is that the spectrum is absolutely continuous, and transport is ultimately ballistic. However, because of the revivals it may take a very long time for the ballistic regime to be reached. This is shown in Fig. 1 for walks starting at the origin. In each case we show two aspects as a function of time, the root mean square deviation of position $\sigma(t)=\left\langle x^{2}\right\rangle^{1 / 2}$ and the probability $p(t)$ of return to the origin. Perfect revivals are identified by the conditions $\sigma(t)=0$, respectively $p(t)=1$. The two fields chosen in the figure are $\Phi_{1}=2 \pi / 5$, for which (5) predicts a fairly weak revival at $t=10(p(10) \geq 0.64)$, which rapidly goes over into ballistic expansion. The envelope of $p(t)$ is well approximated by a Bessel function [41]. The denominator $\Phi_{2}=2 \pi \times 51 / 256$ for the second field is much larger, so the revival predicted by (6) is exponentially sharper: $p(256) \geq 1-10^{-19}$. The full evolution up to $t=256$ therefore repeats roughly $10^{19}$ times, i.e., until the revival errors accumulate sufficiently, to make way for ballistic expansion. This would be true for all fields of the form $\Phi=2 \pi n / 256$, independently of the numerator $n$. However, the evolution up to $t=256$ does depend on the numerator [39]. We can get some information about it from a "rational approximation of the rational" 51/256: Its continued fraction sequence is $\left(c_{0}, c_{1}, c_{2}\right)=(0,5,51)$ and $1 / 5=\Phi_{1} /(2 \pi)$. Therefore, we know that the initial segments of these evolutions coincide. More precisely, from (3) we get $\left\|W_{\Phi_{2}}^{t} \psi-W_{\Phi_{1}}^{t} \psi\right\| \leq t(t+1) / t_{0}^{2}$ with $t_{0} \approx 20$. The comparison for the displayed quantities is even more favorable, as shown in the inset of Fig. 1.

The almost rational case.-The same idea, i.e., combining (3) and (4), can be used with the revival theorem (5), respectively, (6) to get revival estimates for arbitrary irrational $\Phi$ :

$$
\begin{aligned}
\left\|W_{\Phi}^{2 m_{i}} \psi+\psi\right\| & \leq \frac{4 \pi}{c_{i+1}}+\mathcal{O}\left(\frac{L}{m_{i}}\right) \quad m_{i} \text { odd } \\
\left\|W_{\Phi}^{m_{i}} \psi+(-1)^{m_{i} / 2} \psi\right\| & \leq \frac{\pi}{c_{i+1}}+\mathcal{O}\left(\frac{L}{m_{i}}\right) \quad m_{i} \text { even. }
\end{aligned}
$$

That is, if $c_{i_{\alpha}+1} \rightarrow \infty$ along some subsequence $i_{\alpha}$ we get an infinite sequence of sharper and sharper revivals at the corresponding times $m_{i_{\alpha}}$ or $2 m_{i_{\alpha}}$. From each of these revivals we also get a more precise repetition of the entire history up to that point. This shows that the walk operator now has no absolutely continuous spectrum, since for initial vectors in the absolutely continuous subspace the expectation for any finite dimensional projection goes to zero by the Riemann-Lebesgue lemma.

When the sequence $\left(c_{i}\right)$ grows sufficiently fast, we get hierarchical motion on a hierarchy of time scales $m_{i}$, each of which is associated with one approximation $n_{i} / m_{i}$. Sharp revivals, up to errors $\varepsilon_{i}$ with $\varepsilon_{i} \rightarrow 0$, alternate with larger and larger excursions. These are given by intervals $I_{i}$

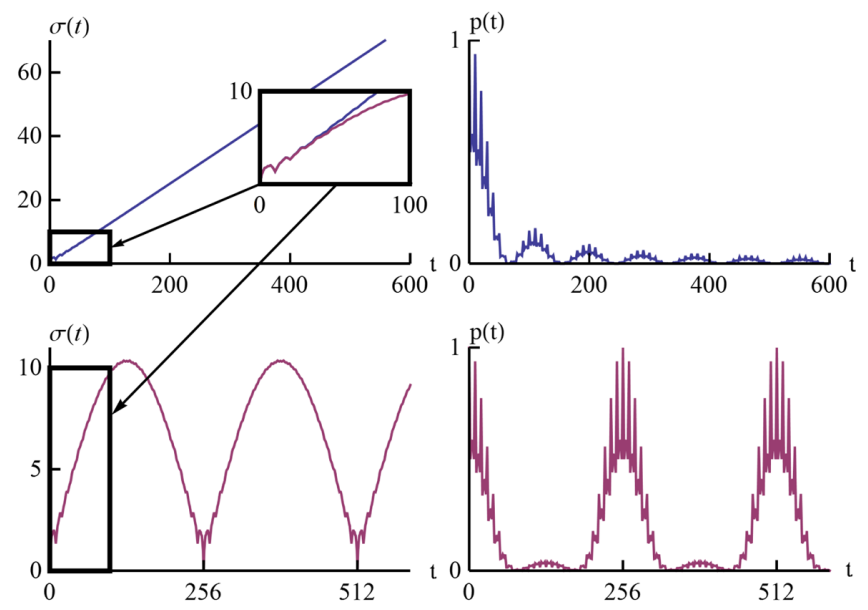

FIG. 1 (color online). Revivals for two walks with rational fields. Top $\Phi_{1}=2 \pi / 5$ and bottom $\Phi_{2}=2 \pi \times 51 / 256$. In both cases the right panel shows the root mean square of the position, and the left panel the probability $p(t)$ to be back at the origin at time step $t$. Since $\Phi_{1}-\Phi_{2} \approx 5 \times 10^{-3}$ the initial segments $(t<100)$ of the top and bottom graphs almost coincide (see black frames and inset). The revivals predicted by the revival theorem are at $t=10$ and $t=256$, respectively. 


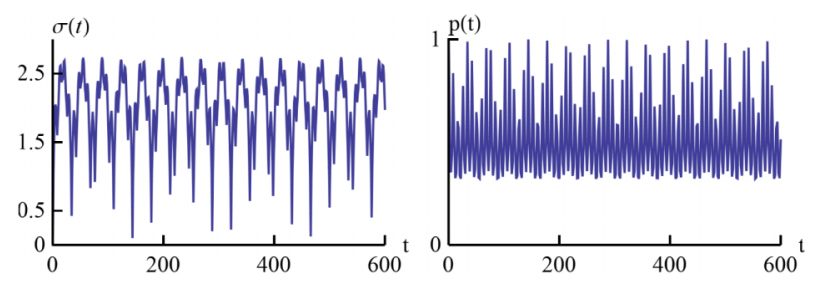

FIG. 2 (color online). Root mean square of position and return probability for the golden ratio field $\Phi=\pi(\sqrt{5}-1)$. There are many returns, and the initial state has a substantial overlap with a bound state, so the return probability is bounded away from zero. Vertical lines: Times appearing in the revival estimate, even though the estimate is trivial.

such that every initial state in $I_{i}$ leaves the interval at the prescribed time with probability close to 1 . The sequences $\varepsilon_{i}$ and $I_{i}$ can be prescribed arbitrarily. This implies singular continuous spectrum. Details of the argument are given in the Supplemental Material [47].

The very irrational case. - Numbers with bounded continued fraction sequence $c_{i}$ do not have exceptionally good rational approximations. For example, for the golden ratio $\varphi$ with $c_{i}=1$ for all $i$, the bound (8) makes no revival predictions at all. Figure 2 shows the behavior of root mean square position and return probability for $\Phi=2 \pi \varphi$. It shows many peaks, not just at the continued fraction denominators, and no tendency for transport or decaying return probability. This is precisely the behavior one would expect from a quantum system with pure point spectrum. Indeed a numerical study readily confirms this guess.

More precisely, we take the system with periodic boundary conditions, diagonalize it, and pick the eigenfunction with the smallest $\left\langle x^{2}\right\rangle$. This eigenfunction decays exponentially (as long as one does not approach the boundary), and converges as the ring size is increased. The same function is obtained by iterating the transfer matrices [43] for the solution of the eigenvalue equation $W_{\Phi} \psi=\exp (i \omega) \psi$. The quasienergy $\omega$ is chosen as $\omega=\Phi / 2$ as this ensures the symmetry of the eigenfunctions around the origin. Therefore, if we start with an arbitrary vector at some very negative site $x=-N$, the iteration will naturally pick the expanding branch, but on passing $x=0$ will decrease again to reach exactly the starting value at $x=+N$. When $N$ is large, this computation has to be carried out with high numerical precision (see Fig. 3). Setting the function to be zero for $|x|>N$ yields an approximate eigenfunction, which solves the eigenvalue equation with a precision (e.g., 2400 digits for the case shown in Fig. 3), which in ordinary numerical work would be considered extraordinarily high.

Knowing one exact eigenfunction is knowing a complete set, since we can generate the required number per site by shifts and staggered sign changes $\psi(x) \mapsto(-1)^{x} \psi(x)$. We emphasize that in spite of the high precision the numerical evaluation does not prove the eigenvalue equation, since
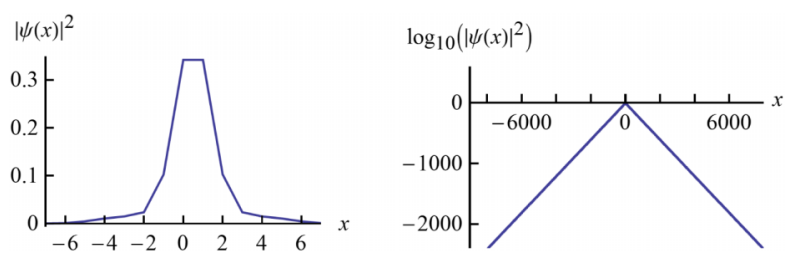

FIG. 3 (color online). Position probability distribution for the eigenfunction of the walk with $\Phi=2 \pi \varphi$. Left: Unscaled plot. Right: Logarithmic plot over the range $[-N, N]$ for which the procedure described in the text was applied. Floating point accuracy for this computation was set to 8000 decimal digits. The slope of the right graph is the inverse Anderson localization length $\approx 0.301$.

for larger $N$ the behavior could be different, and indeed will be different for rationals close enough to $\Phi$. Hence, we have shown Anderson localization only for all practical purposes, meaning for runs with less than $\sim 10^{8000}$ steps, which for nanosecond steps will be incomparably larger than the age of the Universe.

We conjecture in analogy to the case of quasiperiodic potentials in the Hamiltonian setting that localization holds for almost all values of $\Phi$ [48]. In addition to the numerical evidence this is supported by the observation that the localization length is constant, namely, exactly $\log _{10} 2 \approx$ 0.30103 for all irrational fields. This will be subject of future investigations. We note that this conjecture does not contradict the markedly different behavior for rational and almost rational $\Phi$, since these sets are of measure zero.

Summary and outlook.-We have established three typical kinds of longtime behavior of electric quantum walks as a function of the field $\Phi$. An arbitrarily small change in $\Phi$ may change the type of propagation behavior in the infinite time limit. Nevertheless, on a given time scale one can make precise predictions in terms of an initial segment of the continued fraction approximation of $\Phi$. Anderson localization holds for very irrational numbers, and we conjecture that it also holds for random numbers $\Phi$ with probability 1 with a nonrandom localization length. Of the methods developed here, the general approximation technique is relevant to arbitrary quantum walks. The trace formula has analogs for $2 \mathrm{D}$ and 3D quantum walks in magnetic fields (work in progress), also leading to flat bands.

This project was supported by the DFG (Forschergruppe 635), the ERC Grant DQSIM, and NRW Nachwuchsforschergruppe "Quantenkontrolle auf der Nanoskala." A. A. and M. G. also acknowledge support from the Alexander von Humboldt Foundation and the BCGS, respectively.

Note added in proof.-Most recently, Ref. [49] was published, where the authors consider a space and time dependent quantum walk and find in the continuous limit the Dirac equation with an artificial electric field. 
[1] M. Genske, W. Alt, A. Steffen, A. H. Werner, R. F. Werner, D. Meschede, and A. Alberti, Phys. Rev. Lett. 110, 190601 (2013).

[2] S. De Bièvre and G. Forni, J. Stat. Phys. 90, 1201 (1998).

[3] S. Fishman, D. R. Grempel, and R. E. Prange, Phys. Rev. Lett. 49, 509 (1982).

[4] J. Bourgain, Geom. Funct. Anal. 17, 682 (2007).

[5] D. R. Hofstadter, Phys. Rev. B 14, 2239 (1976).

[6] A. Avila and S. Jitomirskaya, Ann. Math. 170, 303 (2009).

[7] G. H. Hardy and E. M. Wright, An Introduction to the Theory of Numbers (Clarendon Press, Oxford, 1998).

[8] R. M. Corless, Am. Math. Mon. 99, 203 (1992).

[9] P. W. Shor, SIAM J. Comput. 26, 1484 (1997).

[10] A. Ambainis, J. Kempe, and A. Rivosh, in Proceedings of the 16th Annual ACM- SIAM Symposium on Discrete Algorithms (SODA'05) (Soc. Indust. Appl. Math., Philadelphia, 2005), p. 1099.

[11] F. Magniez, A. Nayak, J. Roland, and M. Santha, SIAM J. Comput. 40, 142 (2011).

[12] M. Santha, in Proceedings of the Conference on Theory and Applications of Models of Computation (TAMC'08) (Springer, Berlin, 2008), p. 31.

[13] N. B. Lovett, S. Cooper, M. Everitt, M. Trevers, and V. Kendon, Phys. Rev. A 81, 042330 (2010).

[14] A. Patel and K. S. Raghunathan, Phys. Rev. A 86, 012332 (2012).

[15] S. Goswami and P. Sen, Phys. Rev. A 86, 022314 (2012).

[16] A. Ambainis, E. Bach, A. Nayak, A. Vishwanath, and J. Watrous, in Proceedings of the Thirty-Third Annual ACM Symposium on Theory of Computing (STOC '01) (ACM, New York, 2001), p. 37.

[17] G. Grimmett, S. Janson, and P. F. Scudo, Phys. Rev. E 69, 026119 (2004).

[18] A. Ahlbrecht, C. Cedzich, R. Matjeschk, V. Scholz, A. Werner, and R. Werner, Quantum Inf. Process. 11, 1219 (2012).

[19] A. Ahlbrecht, H. Vogts, A. H. Werner, and R. F. Werner, J. Math. Phys. (N.Y.) 52, 042201 (2011).

[20] P. Kurzyński and A. Wójcik, Phys. Rev. Lett. 110, 200404 (2013).

[21] M. Mohseni, P. Rebentrost, S. Lloyd, and A. AspuruGuzik, J. Chem. Phys. 129, 174106 (2008).

[22] O. Mülken, V. Bierbaum, and A. Blumen, J. Chem. Phys. 124, 124905 (2006).

[23] O. Mülken and A. Blumen, Phys. Rev. E 71, 036128 (2005).

[24] A. Regensburger, C. Bersch, B. Hinrichs, G. Onishchukov, A. Schreiber, C. Silberhorn, and U. Peschel, arXiv:1104.0105.

[25] P. Kurzyński, Phys. Lett. A 372, 6125 (2008).

[26] T. Kitagawa, M. S. Rudner, E. Berg, and E. Demler, Phys. Rev. A 82, 033429 (2010).

[27] T. Kitagawa, Quantum Inf. Process. 11, 1107 (2012).
[28] A. Ahlbrecht, A. Alberti, D. Meschede, V. B. Scholz, A. H. Werner, and R. F. Werner, New J. Phys. 14, 073050 (2012).

[29] M. Karski, L. Förster, J. M. Choi, W. Alt, A. Widera, and D. Meschede, Phys. Rev. Lett. 102, 053001 (2009).

[30] F. Zähringer, G. Kirchmair, R. Gerritsma, E. Solano, R. Blatt, and C.F. Roos, Phys. Rev. Lett. 104, 100503 (2010).

[31] H. Schmitz, R. Matjeschk, C. Schneider, J. Glueckert, M. Enderlein, T. Huber, and T. Schaetz, Phys. Rev. Lett. 103, 090504 (2009).

[32] A. Peruzzo, M. Lobino, J. C. Matthews, N. Matsuda, A. Politi, K. Poulios, X. Q. Zhou, Y. Lahini, N. Ismail, K. Worhoff, Y. Bromberg, Y. Silberberg, M. G. Thompson, and J. L. OBrien, Science 329, 1500 (2010).

[33] L. Sansoni, F. Sciarrino, G. Vallone, P. Mataloni, A. Crespi, R. Ramponi, and R. Osellame, Phys. Rev. Lett. 108, 010502 (2012).

[34] A. Schreiber, K. N. Cassemiro, V. Potoček, A. Gábris, P. J. Mosley, E. Andersson, I. Jex, and C. Silberhorn, Phys. Rev. Lett. 104, 050502 (2010).

[35] A. Schreiber, A. Gabris, P.P. Rohde, K. Laiho, M. Štefaňák, V. Potocek, C. Hamilton, I. Jex, and C. Silberhorn, Science 336, 55 (2012).

[36] M. A. Broome, A. Fedrizzi, B. P. Lanyon, I. Kassal, A. Aspuru-Guzik, and A. G. White, Phys. Rev. Lett. 104, 153602 (2010).

[37] M.S. Underwood and D.L. Feder, Phys. Rev. A 85, 052314 (2012).

[38] A. M. Childs, D. Gosset, and Z. Webb, Science 339, 791 (2013).

[39] M. C. Bañuls, C. Navarrete, A. Pérez, E. Roldán, and J. C. Soriano, Phys. Rev. A 73, 062304 (2006).

[40] O. Buerschaper and K. Burnett, arXiv:quant-ph/0406039.

[41] A. Wójcik, T. Łuczak, P. Kurzyński, A. Grudka, and M. Bednarska, Phys. Rev. Lett. 93, 180601 (2004).

[42] P. W. Anderson, Phys. Rev. 109, 1492 (1958).

[43] A. Ahlbrecht, V. B. Scholz, and A.H. Werner, J. Math. Phys. (N.Y.) 52, 102201 (2011).

[44] A. Aspect and M. Inguscio, Phys. Today 62, No. 8, 30 (2009).

[45] M. Reed and B. Simon, Methods of Modern Mathematical Physics (Academic Press, New York, 1980), Vol. 1.

[46] G. Grimmett, S. Janson, and P. F. Scudo, Phys. Rev. E 69, 026119 (2004).

[47] See Supplemental Material at http://link.aps.org/ supplemental/10.1103/PhysRevLett.111.160601 for the proof of the revival theorem and an explicit construction of the hierarchical motion.

[48] J. Bourgain and M. Goldstein, Ann. Math. 152, 835 (2000).

[49] G. Di Molfetta, M. Brachet, and F. Debbasch, arXiv:1309.4923. 